\title{
Aproximación a La Caracterización Ecológica de La Quebrada Fucha en La Reserva Forestal de los Cerros Orientales en La Localidad de San Cristóbal. (Bogotá, Colombia)
}

\section{Approximation of the Ecological Characterization of Fucha Stream in the Forest Reserve of the East Hills In San Cristóbal Locality (Bogotá, Colombia)}

Por: Restrepo Villamil Mónica; Rincón Rincón Claudia Marcela ${ }^{1}$

Resumen: En este trabajo se presentan los resultados obtenidos en la práctica de campo realizada el día 10 de octubre de 2008, en la quebrada Fucha en una reserva forestal protegida por la Empresa de Acueducto y Alcantarillado de Bogotá en los cerros orientales de la localidad de San Cristóbal. El objetivo principal de este estudio fue realizar una aproximación a la caracterización de este sistema acuático, para lo cual, se colectaron insectos acuáticos (entomofauna) y perifiton, se caracterizaron los suelos de la ribera y se realizaron análisis de las variables fisicoquímicas de la zona. Los insectos acuáticos que se encontraron sumaron un total de 188 individuos, en donde las familias más abundantes fueron Simuliidae y Ceratopogonidae con 60 individuos cada una $(32 \% \mathrm{c} / \mathrm{u})$ y las de menor abundancia fueron Odontoceridae, Lepharoceridae y Pyralidae con 1 individuo c/u $(0.53 \% \mathrm{c} / \mathrm{u})$; por otra parte, se encontraron 14 géneros de perifiton. El sistema presenta una diversidad media de acuerdo al índice de Shannon (1.55). La principal fuente de energía del sistema es la materia orgánica disuelta, por lo que la vía que predomina es la detrítica.

Palabras Clave: Insectos acuáticos, flujos de energía, sistema acuático, variables fisicoquímicas.

Abstract: In this paper we present the obtained results from the field practicum carried out on October 10th 2008, in Fucha stream in a forest reserve protected by the Supply and Sewerage Company of Bogotá, in the east hills of San Cristobal locality. The main objective of this study was to make an approach to the characterization of this aquatic system, for which aquatic insects and periphyton were collected, soils along the river were and an

${ }^{1}$ Estudiantes de Licenciatura en Biología. Universidad Pedagógica Nacional. ialacon1227@yahoo.es, gmonik8@gmail.com 
analysis of the physical-chemical and hydrological variables of the area was. carried out.

The aquatic insects that were found came to a total of 188 individuals, where the most abundant families were Simuliidae and Ceratopogonidae with 60 individuals each one of them $(32 \%)$ and the ones that had the lowest abundance were Odontoceridae, Lepharoceridae and Pyralidae with an individual each one $(0.53 \%)$, on the other hand, 14 genera of periphyton were found. The system has an average diversity range according to the index of Shannon (1.55). The main source of energy in the system is the dissolved organic matter, thus the predominant route is the detrital.

Key Words: Aquatic insects, energy flows, aquatic system, physicochemical variables.

\section{INTRODUCCIÓN}

Este estudio constituye una primera aproximación a la caracterización ecológica de un sistema acuático como lo es la quebrada Fucha. Este sistema se encuentra en una zona bajo el cuidado de la Empresa de Acueducto y Alcantarillado de Bogotá, pese a lo anterior se evidencia un alto grado de intervención antrópica que se inicia en la zona alta de este sistema fluvial y lo cual resulta preocupante ya que el río Fucha es el más importante de la ciudad, porque se encuentra en contacto con un mayor número de localidades (11), convirtiéndose en el corazón hídrico de Bogotá y uno de los que mayor riesgo tiene su estructura ecológica principal (CORFO y DAMA, 2003).

El río Fucha nace a $3500 \mathrm{msnm}$ en la estrella fluvial del páramo de Cruz Verde, su recorrido de 21.7 kilómetros comienza por el oriente hacia el occidente donde se encuentra con sus afluentes: río Palo Blanco, quebrada La Mirla, quebrada La Osa, quebrada Fotoga, quebrada La Upatá, quebrada La Plazuela, quebrada La Colorada, quebrada El Carral y quebrada El Soche (Lanthon, 2003).

A nivel hidrólogico, de acuerdo con los datos obtenidos de la estación El Delirio de la Empresa de Acueducto y Alcantarillado de Bogotá, este río en época de altas precipitaciones (período en el que se realizo el estudio), presenta un caudal medio multianual para el mes de octubre de $0.570 \mathrm{~m}^{3} / \mathrm{s}$ y un promedio multianual de $0.649 \mathrm{~m}^{3} / \mathrm{s}$ aproximadamente, y el caudal promedio calculado en la zona fue de $2.28 \mathrm{~m}^{3} / \mathrm{s}$ (Lanthon, 2003).

Así mismo, la importancia de este río radica en que, además de ancestral, tiene que ver con la historia del desarrollo de la ciudad. Esta cuenca en donde se construyó el primer acueducto que tuvo la ciudad llamado Vitelma, recibe en la parte alta el nombre de Río San Cristóbal y a partir de la avenida caracas Río Fucha.

Desde entonces, la cuenca de este río es víctima de la invasión de su ronda, la contaminación de sus aguas por todo tipo de vertimientos, especialmente los industriales, el arrojo al caudal de basuras, escombros, destrucción de las especies de fauna y flora, canalización del cauce, secamiento e invasión de los humedales y la ejecución de obras 
civiles anti-técnicas en su ronda (CORFO, 2003). Lo anterior resalta la importancia del presente estudio, para incentivar desde la escuela y más específicamente desde el área de ciencias naturales y biología, el cuidado de este tipo de ecosistemas de alta montaña ya que poseen importantes recursos hídricos para los centros urbanos.

Los ríos y los ecosistemas terrestres que componen la cuenca vertiente mantienen numerosas interrelaciones. Los regímenes de caudales, sedimento, nutrientes y la materia orgánica originados en las laderas de la cuenca conforman los hábitats físicos del río, suministrando energía y recursos a las comunidades. En especial, la vegetación de las riberas estabiliza las orillas del cauce, contribuyendo con materiales leñosos y residuos vegetales a la materia orgánica del río y controlan la entrada de luz y la llegada de otras materias orgánicas y nutrientes al cauce (Botero, 2004).

La cuenca de un río se caracteriza por su cubierta vegetal, geología, topografía, suelos y su uso. Por esto, para lograr una aproximación cercana al sistema acuático es necesario hacer un estudio global de estas características y su influencia en el río y las comunidades bióticas que en él habitan (Margalef, 1983).

Los ríos constituyen sistemas abiertos y heterotróficos, pues el aporte más importante de energía al sistema procede de las orillas del arroyo o riberas, las cuales poseen una enorme superficie de interacción con el sistema acuático. Este material aportado sufre la acción de diversos organismos, como es el caso de los insectos acuáticos (Roldán, 1992).

El cauce, queda definido longitudinalmente por el espacio ocupado por el río desde su nacimiento hasta su desembocadura, respecto a su dimensión transversal se tiene en cuenta el nivel que alcanzan las aguas y la frecuencia de las inundaciones que determina la presencia de un tipo u otro de vegetación. El trazado de los ríos hace referencia a la trayectoria que desarrollan, entre los que se encuentran trazado recto (sinuosidad inferior a 1.5), meandriforme (sinuosidad superior a 1.5) y trenzado (en tramos de mayor pendiente y carga sólida) (González, 1998).

Los sistemas acuáticos de alta montaña se caracterizan por su calidad físico química del agua generalmente buena. El oxígeno disuelto es alto, las aguas son claras y transparentes, pobres en nutrientes y de baja conductividad; la producción primaria es muy baja (Botero, 2004).

El oxígeno disuelto es uno de los gases más importantes en los ecosistemas acuáticos; su presencia y concentración definen el tipo de especies que ocurren de acuerdo a sus tolerancias y rangos de adaptación, por lo que establecen toda la estructura y funcionamiento biótico de los ecosistemas (Ramírez, 1998). La fuente principal de oxígeno es el aire, que se difunde rápidamente en el agua por la turbulencia (Roldán, 2003).

Los insectos acuáticos se agrupan en 10 órdenes para Colombia, los cuáles constituyen la fauna más representativa de lagos y ríos (Roldán, 1992). El trabajo con estos organismos permite evaluar las características de un sistema acuático ya que se emplean como bioindicadores cuya presencia y abundancia señalan algún proceso o estado del sistema acuático (Pinilla, 1998) y, gracias a su tamaño relativamente grande y a sus ciclos de 
desarrollo de fácil manejo para esta clase de estudios; la diversidad que presentan da cuenta de un amplio rango de tolerancia frente a diferentes parámetros de contaminación (Hellawell, 1986) además, su distribución espacial, abundancia y diversidad se encuentran estrechamente relacionadas con las variables hidrológicas y físico-químicas.

Los insectos acuáticos permiten pautar la red de relaciones que se establecen en un sistema acuático, ya que permiten comprender las características de un cuerpo de agua en términos de diversidad de organismos presentes, calidad del agua y variables fisicoquímicas (Merrit, 1996).

De acuerdo a la clasificación de Cummins y Klug (1979) de los diferentes recursos alimenticios que utilizan los insectos acuáticos en los ríos, se ubican como una de las fuentes de energía para estos sistemas a los detritos o Materia Orgánica Particulada (MOP), ésta a su vez se agrupa en: Materia Orgánica Particulada Gruesa (MOPG) que incluye hojas, troncos, flores y otros materiales en descomposición (hojarasca), Materia Orgánica Particulada Fina (MOPF) como material vegetal que ya ha sido descompuesta y Materia Orgánica Disuelta (MOD) principalmente ácidos fulvicos y húmicos, cuya descomposición provee de iones al sistema (Merrit, 1996).

Sin embargo, centrándose en la productividad del sistema lo óptimo es considerar simultáneamente su funcionamiento y estructura. Ello se facilita mediante la clasificación de los organismos en grupos funcionales (Cummins, 1975) al relacionar directamente los componentes biológicos con los procesos de flujos energéticos. Los miembros de cada grupo funcional realizan un proceso similar: los productores primarios (perifiton, fitoplancton y macrófitas) fijan la energía del sol; hongos y bacterias descomponen la materia orgánica; y los grupos funcionales consumidores incluyen principalmente a insectos acuáticos y peces, que utilizan la energía acumulada por los grupos anteriores.

Los consumidores a su vez, se agrupan según el tipo de alimento y según los mecanismos que utilizan en su alimentación: a) los desmenuzadores se alimentan de partículas grandes de detritos orgánicos (hojas, ramillos, restos de animales, macrófitas); los fitófagos se alimentan de perifiton mediante el raspado de la superficie que ocupan, o del fitoplancton mediante filtración; c) los colectores filtran las partículas finas de detritus suspendidas en la columna de agua, o bien, recolectan las sedimentadas; y los depredadores y parásitos obtienen sus alimentos de otros animales (Santander, 2007) (Merrit, 1996).

A partir de lo anterior, se puede establecer un modelo de flujo de energía que permite comprender que en un sistema lótico existe una dinámica que se sustenta en las interacciones que se establecen entre los diferentes componentes tanto internos como externos al sistema.

Dentro de los estudios realizados acerca del tema se destaca el de Lanthon (2003), quien realiza una caracterización de insectos acuáticos en los ríos Arzobispo y San Cristóbal en los cerros orientales en Bogotá (Colombia), donde se encuentran 31 clases cuyas abundancias abarcan desde 1 hasta 12464 individuos en la familia Chironomidae del orden diptera. 
Teniendo en cuenta lo anterior, el objetivo de este trabajo es lograr una aproximación a la caracterización ecológica de la Quebrada Fucha en la reserva Forestal de los cerros orientales en la Localidad de San Cristóbal, Bogotá (Colombia).

\section{MATERIALES Y MÉTODOS}

El trabajo se desarrolló en la quebrada Fucha a una altitud de 3000 msnm, latitud: $04^{\circ} 31^{\prime} \mathrm{N}$ longitud: $74^{\circ} 03^{\prime} \mathrm{W}$; este sistema se caracteriza por ser montañoso con pendientes de $10^{\circ} \mathrm{a}$ $15^{\circ}$ de inclinación en la zona de estudio (Fotografía 1). Se realizó un solo muestreo el día 10 del mes de octubre de 2008, donde se tomo una muestra compuesta de suelo a partir de tres submuestras, aproximadamente de $1 \mathrm{~kg}$ de peso, evaluándose en el laboratorio la capacidad de campo, $\mathrm{pH}$, grado de saturación, peso, textura y color.

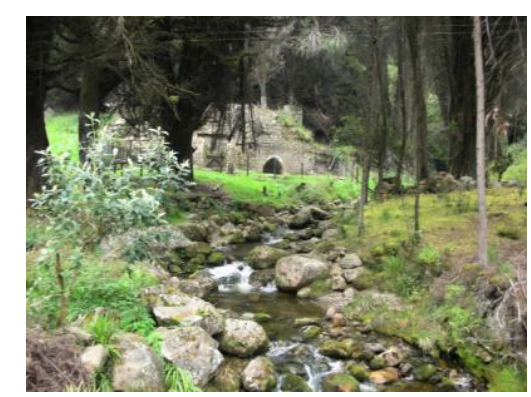

Fotografía 1. Zona de muestreo. Tomada por: Mónica Restrepo Villamil

Se evaluaron variables hidrológicas tales como: amplitud del cauce en tres zonas, amplitud de la zona de inundación, la profundidad cada $20 \mathrm{~cm}$ y velocidad de corriente. De igual manera se analizaron algunos parámetros fisicoquímicos como: turbidez (Turbidímetro Hanna Hi 9370), conductividad - pH (pHmetro Mi 805 pH/EC/TDS/Temperature Portable), nitritos - amonio- alcalinidad - cloruros (kits de Merk), temperatura del agua y sólidos totales (Oxímetro VSI No. 55/50 FT Serial 99C1415).

También se colectaron insectos acuáticos bajo la técnica de coriotopos (Rincón, 1996), los sustratos seleccionados fueron, piedra corriente lenta (PCL), piedra corriente rápida (PCR), cascada (CAS), gravilla (GR), musgo en roca (MR) y ribera (RIB). Los organismos colectados se preservaron en alcohol etílico al $70 \%$ y se determinaron hasta familia en todos los casos empleando las claves propuestas por Roldán (1988).

De otro lado, se realizó la valorización del sistema ripario a partir del índice de calidad de QBR, que emplea los siguientes parámetros: cobertura de la vegetación, estructura de la cobertura, calidad de la cobertura y alteración del canal.

Posteriormente, se limpiaron las muestras en el laboratorio de la Universidad Pedagógica Nacional, donde se caracterizaron los aparatos bucales y contenidos intestinales de los insectos acuáticos teniendo en cuenta la técnica de Merrit (1996), lo anterior sumado las características del ecosistema, el tipo de entomofauna encontrada y la ayuda de referentes bibliográficos, permitió hacer una aproximación de los hábitos tróficos y el flujo de energía del sistema. 
Para el muestreo del perifiton, se empleo la técnica propuesta por Loewe - Laliberte (1996), donde se realizó un lavado - raspado de la superficie de 30 rocas medianas con un cepillo de cerdas suaves. Para la identificación taxonómica se emplearon las claves propuestas por Bicudo (1970).

\section{RESULTADOS Y DISCUSIÓN}

\section{Variables hidrológicas}

El balance hídrico de la zona, (Figura 1) obtenido con los datos del último año de la estación El Delirio de la Empresa de Acueducto y Alcantarillado de Bogotá, permite comprender la dinámica del río a lo largo del año y en especial el comportamiento hídrico durante el estudio; se observan deficiencias de agua en los meses de enero a febrero y principios de marzo, con una recarga en los meses de marzo a julio, un exceso de la misma de julio a septiembre, una nueva época de recarga de septiembre a noviembre y un exceso de agua de noviembre a diciembre.

Cabe resaltar que los valores de precipitación se muestran casi todo el año por encima de los de evapotranspiración (ETP), lo cual indica que esta zona presenta casi durante todo el año exceso de agua, y por consiguiente elevados valores de humedad, hecho que se contrasta con el tipo de vegetación presente (alta cantidad de epífitas).

En cuanto al suelo, se determinó una textura de tipo franco arcillo arenosa y arenosa franca, que se caracterizan por poseer un alto grado de rugosidad.

Respecto al pH del suelo, se encontró un promedio de las tres submuestras de 4.6, que corresponden a suelos de tipo ácido como resultado de los ácidos propios de la lluvia, formados en la atmósfera por el dióxido de azufre (SO2) y óxidos de nitrógeno aportados principalmente por la quema de combustibles fósiles; y de la concentración de arcilla que el suelo posee. Se considera que el valor de $\mathrm{pH}$ obtenido se acentúa como resultado de la perturbación aledaña al área de estudio (cultivo de truchas), que aporta materia orgánica al sistema con un efecto acidificante en el agua y el suelo.

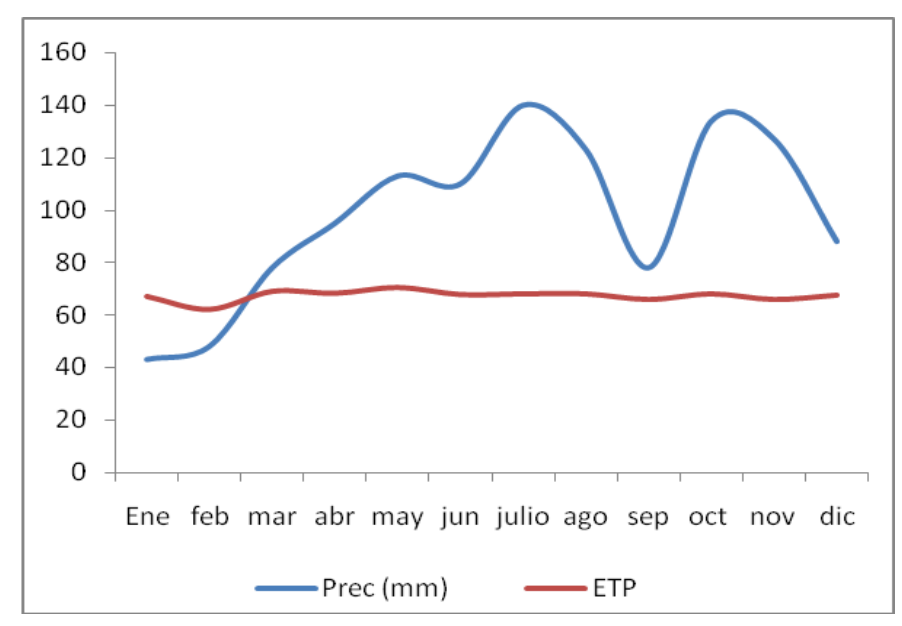

Figura 1. Balance Hídrico de la zona de estudio. Datos suministrados por la estación El Delirio de la Empresa de Acueducto y Alcantarillado de Bogotá. 
La estructura del suelo se estableció dentro de la categoría granular con gran aporte de materia orgánica, hifas de hongos, raíces finas y excreciones (Zapata, 2006).

La capacidad de campo del suelo fue de $85.7 \%$, un valor relativamente alto que se correlaciona con la textura predominante en los suelos anteriormente descrita, la cual se caracteriza por poseer una moderada concentración de arcilla que permite este porcentaje de retención de agua por parte del suelo.

Por otra parte, el suelo tiende a una coloración parda que puede deberse a la mayor cantidad de materiales húmicos.

Los valores de amplitud del cauce en promedio de las tres zonas es de $5.84 \mathrm{~m}$ con una profundidad mínima de $2 \mathrm{~cm}$ y una máxima de $33.5 \mathrm{~cm}$, esta última se encuentra relacionada con la zona de rápidos. La zona de inundación, para la zona A del estudio no es muy amplia con valores en la orilla derecha $0.40 \mathrm{~m}$ y orilla izquierda $0.50 \mathrm{~m}$, para la zona B con valores en la orilla derecha $0.80 \mathrm{~m}$ y orilla izquierda $0.52 \mathrm{~m}$ y en la zona C con una zona de inundación más amplia en la orilla derecha con $1.04 \mathrm{~m}$ y orilla izquierda $0.54 \mathrm{~m}$.

Dentro de la caracterización del sistema, se estableció que el tipo de corriente que presenta la quebrada es permanente con una pendiente de $15^{\circ}$, transportando lentamente partículas como cantos rodados, guijarros y gravas, el tamaño de las partículas (entre $1.5-1.1 \mathrm{~cm}$ y $25.3-16.5 \mathrm{~cm}$ ) (Tabla 1) y la baja temperatura del lugar, permite inferir que el flujo que presenta la quebrada es turbulento (Botero, 2004). Los sólidos disueltos superficiales (0.001gr) y del lecho del río (0.003 gr), sumado a la ubicación geográfica y al perfil topográfico realizado del lugar, indican que el sistema en el área del estudio es de primer orden, ya que no ha recibido aguas aún de ningún tributario (CORFO, 2003) (Roldán, 1992).

\begin{tabular}{|c|}
\hline GRANULOMETRÍA \\
\hline $9.2 \mathrm{~cm}-3.2 \mathrm{~cm}$ \\
\hline $3.6 \mathrm{~cm}-8.2 \mathrm{~cm}$ \\
\hline $7.1 \mathrm{~cm}-4.5 \mathrm{~cm}$ \\
\hline $5.2 \mathrm{~cm}-2.1 \mathrm{~cm}$ \\
\hline $6.8 \mathrm{~cm}-4.3 \mathrm{~cm}$ \\
\hline $3.6 \mathrm{~cm}-5.5 \mathrm{~cm}$ \\
\hline $4.5 \mathrm{~cm}-3.3 \mathrm{~cm}$ \\
\hline $6.3 \mathrm{~cm}-3.6 \mathrm{~cm}$ \\
\hline
\end{tabular}




\begin{tabular}{|c|}
\hline $9.8 \mathrm{~cm}-15.4 \mathrm{~cm}$ \\
\hline $25.3 \mathrm{~cm}-16.5 \mathrm{~cm}$ \\
\hline $15.6 \mathrm{~cm}-9.3 \mathrm{~cm}$ \\
\hline $12.5 \mathrm{~cm}-5.4 \mathrm{~cm}$ \\
\hline $2.5 \mathrm{~cm}-1.3 \mathrm{~cm}$ \\
\hline $1.6 \mathrm{~cm}-1.6 \mathrm{~cm}$ \\
\hline $1.5 \mathrm{~cm}-1.1 \mathrm{~cm}$ \\
\hline
\end{tabular}

Tabla 1. Datos de Granulometría

\section{Variables biológicas}

En el muestreo realizado se encontró un total de 188 individuos, distribuidos en diez (10) familias pertenecientes a cuatro (4) órdenes, siendo el orden Díptera el más abundante con 161 individuos, seguido del orden Ephemeroptera con 17 individuos. El orden menos abundante fue Lepidóptera con 1 individuo.

Las familias Simuliidae y Ceratopogonidae fueron las más representativas con un $32 \%$ cada una; seguidas de la familia Chironomidae con 21.2\%; cabe resaltar que las familias Odontoceridae, Lepharoceridae y Pyralidae fueron las menos abundantes con $0.53 \%$.(Figura 2).

El coriotopo que presentó la mayor abundancia fue el musgo con $39 \%$, seguido por PCR con $24 \%$, el que mostró menos individuos fue gravilla con $1.6 \%$ (Figura 3).

El índice de Shannon arrojo un valor de 1.55 bit/ind, lo que indica un alto grado de uniformidad; la diversidad para los insectos acuáticos se catalogó como media (1.7 bits/ind.), se presenta poca abundancia frente a una alta equitatividad (0.67), este último indica una alta similaridad de las familias encontradas en la zona. Se estima que la riqueza es media, dado el número de familias encontradas (10) en esta quebrada.

El valor arrojado por el índice de Simpson (0.25) entre las familias de insectos acuáticos, da cuenta de una baja dominancia, sin embargo, las familias Simullidae y Ceratopogonidae fueron las dominantes. 


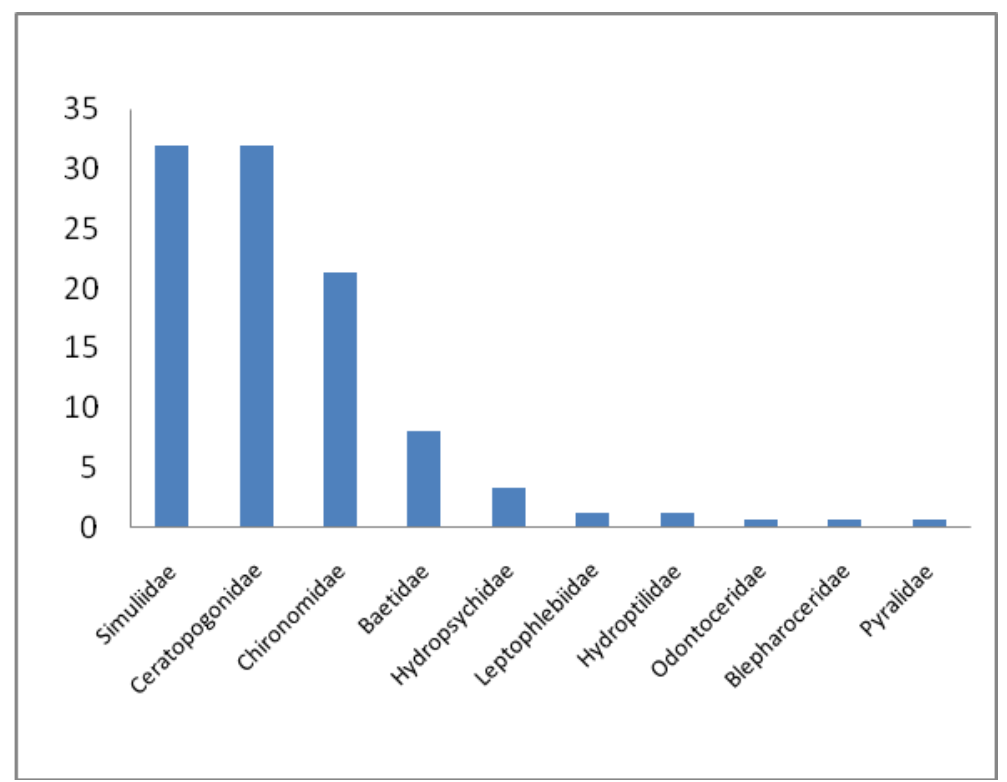

Figura 2. Abundancia relativa de insectos acuáticos de acuerdo a la familia.

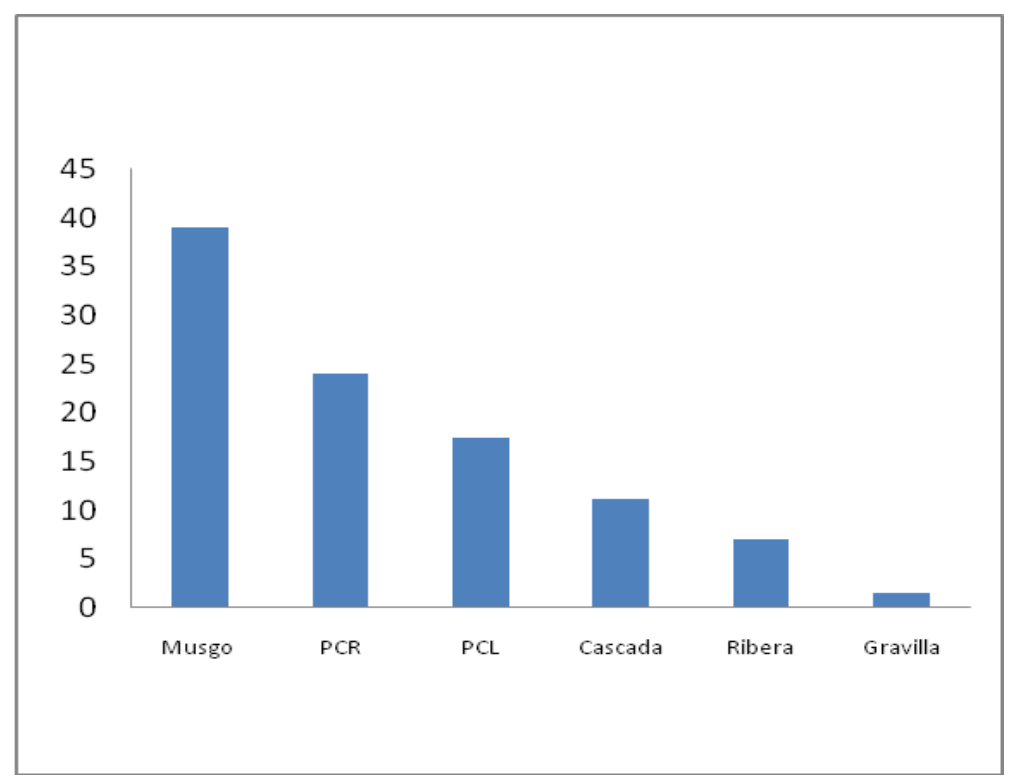

Figura 3. Abundancia de insectos acuáticos por coriotopo.

En cuanto a la comunidad perifítica se encontraron catorce (14) géneros (Tabla 2), entre estos se encuentran Anabaena, Trachelomona y Oscillatoria (Donato, 1996).

\begin{tabular}{|c|}
\hline Género \\
\hline Anabaena \\
\hline Cosmarium \\
\hline
\end{tabular}




\begin{tabular}{|c|}
\hline Eunotia \\
\hline Mellosyra \\
\hline Mougeotia \\
\hline Navicula \\
\hline Nodularia \\
\hline Oedogonium \\
\hline Oscillatoria \\
\hline Pinnularia \\
\hline Spyrogira \\
\hline Trachelomona \\
\hline Ulotrix \\
\hline
\end{tabular}

Tabla 2. Reporte de perifíton

\section{Vegetación}

La vegetación riparia encontrada presenta una estratificación arbórea, arbustiva y herbácea, siendo el estrato más representativo el arbustivo. El grado de desgaste de la ribera es de baja susceptibilidad, puesto que se encuentra protegida por una red de raíces, partículas resistentes grandes (como las rocas) y un ángulo suave en cuanto a la pendiente $\left(15^{\circ}\right)$.

Los árboles de la ribera dan sombra a gran parte del cauce (con una cobertura del 65\%), por lo que la entrada de energía lumínica es escasa.

En la quebrada Fucha, los recursos materiales y energéticos del sistema están representados por los nutrientes inorgánicos y diversos tipos de materia orgánica. El material leñoso aportado por los árboles constituye una fuente no solo de recursos energéticos sino que provee una gran variedad de refugios para la vida en la zona (Botero, 2004).

El QBR dio un valor total para la zona de muestreo de 45, que hace referencia a que el sistema presenta una fuerte alteración antrópica expresada en viviendas, basuras y cultivo de truchas. El mayor aporte natural al sistema de materia orgánica proviene de la vegetación por lo que se considera como una fuente importante de energía.

Cabe resaltar la alta presencia de briofitos, especialmente en los troncos caídos. Se presenta vegetación característica de zonas húmedas tales como quiches, helechos y musgos (Fotografía 2a y b).

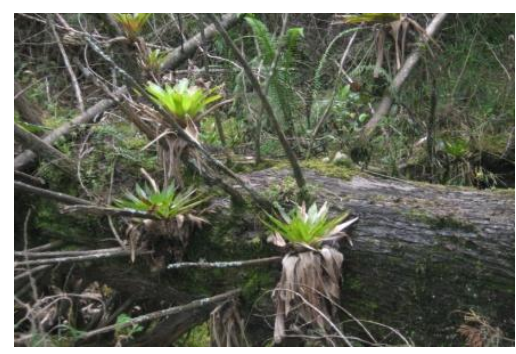




\section{Fotografía 2. a. Epífitas del área de estudio. Tomada por: Claudia Marcela Rincón Rincón.}

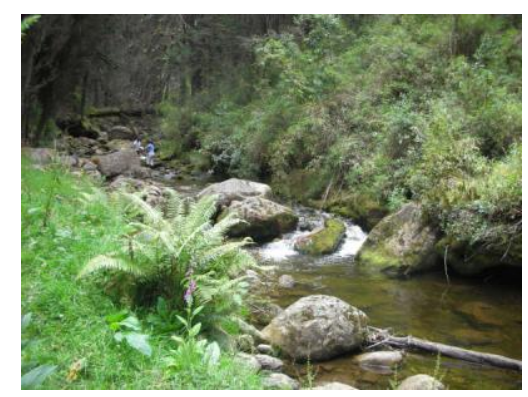

Fotografía 2. b. Musgos y helechos del área de estudio. Tomada por: Claudia Marcela Rincón Rincón.

\section{Variables físico-químicas}

En este estudio, el valor registrado de oxígeno corresponde a $6.71 \mathrm{mg} / \mathrm{L}$; con una temperatura del agua de $8.05^{\circ} \mathrm{C}$, esta temperatura genera una diferencia de densidades lo que promueve una mezcla de este gas en el agua al aumentar su solubilidad, esto a su vez, se relaciona con el caudal de la quebrada, la descarga y la altitud a la que se encuentra el sistema (Roldan, 1992). Estos valores constituyen las condiciones adecuadas para el desarrollo de organismos que requieren aguas limpias y con altas concentraciones de oxígeno, (Ramírez, 1998) lo que coincide con las abundancias de las familias Simuliidae y Ceratopogonidae que necesitan altos niveles de oxígeno disuelto y en general aguas limpias o con poco grado de contaminación.

El pH (6.66) muestra que el sistema es ligeramente ácido con tendencia a la neutralidad, producido bajo condiciones de oxidación de materia orgánica con liberación de $\mathrm{CO}_{2}$ (Ramírez, 1998) o por la incorporación de sales ácidas por parte de la lluvia, el cultivo de truchas y las características típicas de este tipo de suelos de la región andina que tiene orígenes volcánicos que contribuyen con los valores de $\mathrm{pH}$ ácidos o ligeramente ácidos; esto relacionado con los valores de alcalinidad parcial $(0.55 \mathrm{mg} / \mathrm{l} \mathrm{CaCO}$ ) encontrados, muestran la capacidad del agua para neutralizar los ácidos presentes que se relacionan con los niveles de $\mathrm{CO}_{2}$.

El valor de $\mathrm{pH}$ obtenido se encuentra dentro de los rangos límites descritos por Machado y Roldán (1981) para la supervivencia de la mayoría de los organismos acuáticos, que varían entre 4.5 y 8.5 unidades. Lo que vendría a explicar la baja abundancia de los organismos muestreados.

Este conjunto de variables contribuyen a establecer que la conductividad, que hace referencia a la capacidad del agua de conducir la corriente eléctrica en función de los iones 
presentes (Wetzel, 1979), es baja $(42 \mu \mathrm{S} / \mathrm{cm})$ con bajo grado de mineralización; situación que se apoya en los valores de cloruros $(0.45 \mathrm{mg} / \mathrm{L})$ hallados, si se tiene en cuenta que éstos son algunos de los principales causantes de dicha propiedad (Tabla 3 ).

\begin{tabular}{|c|c|}
\hline \multicolumn{2}{|c|}{ Características Fisicoquímicas } \\
\hline Temperatura & $8.05{ }^{\circ} \mathrm{C}$ \\
\hline Oxígeno & $6.71 \mathrm{mg} / \mathrm{L}$ \\
\hline Turbidez & $1.64 \mathrm{FTU}$ \\
\hline $\begin{array}{c}\text { Alcalinidad } \\
\text { total }\end{array}$ & $0.55 \mathrm{mg} / \mathrm{l} \mathrm{CaCO} 3$ \\
\hline $\begin{array}{c}\text { Nitritos } \mathrm{NH}^{+4} \\
\left(\mathrm{NO}_{2}\right)\end{array}$ & Cercano a cero \\
\hline $\mathrm{pH}$ & 6.66 \\
\hline Cloruros & $0.45 \mathrm{mg} / \mathrm{L}$ \\
\hline Nitratos & $25 \mathrm{mg} / \mathrm{L} \mathrm{NO} 3$ \\
\hline Sólidos totales & $8 \mathrm{mg} / \mathrm{L}$ \\
\hline Amonio & 0 \\
\hline
\end{tabular}

Tabla 3. Características Fisicoquímicas de la quebrada Fucha

La turbidez se puede establecer como una medida de los sólidos disueltos totales presentes en el sistema, para el caso de la zona de estudio es bajo (1.64 FTU), lo que se evidencia en la transparencia de este cuerpo de agua. El valor de los sólidos disueltos $(11.5 \mathrm{mg} / \mathrm{L})$, puede ser resultado del arrastre superficial, influenciados por el caudal del río $\left(0.649 \mathrm{~m}^{3} / \mathrm{s}\right)$ y la pendiente de la zona de estudio $\left(25^{\circ}\right)$, haciendo que los sedimentos sean continuamente arrastrados y se mantengan suspendidos, evitando que una gran proporción se deposite en el lecho de la quebrada.

En lo referente a los valores de nitritos estos son cercanos a cero, lo cual explica la poca concentración de iones que puedan establecer enlaces con otros presentes en la ribera y así generar el arrastre de dichos materiales.

\section{Flujo de Energía}

En principio, la energía ingresa a la quebrada con la conversión de la energía lumínica en energía química.

A este flujo de energía (Anexo 1) se le suman dos entradas más al ecosistema, la primera corresponde al material alóctono como MOPF (Materia Particulada Fina) y MOPG (Materia Orgánica Particulada Gruesa), producto de la vegetación riparia de la zona.

El material alóctono que cae de los árboles o que es arrastrado por la lluvias (Roldan, 1992) y los residuos aportados por el cultivo de truchas, se descompone lentamente por acción de bacterias y hongos que poseen una tasa alta de renovación del sistema, dando lugar a detritus en forma de "partículas gruesas" de materia orgánica, que son aprovechadas por 
los consumidores de la quebrada en ese tramo (Botero, 2004). La rapidez con que se descompone para ser aprovechada por los diferentes organismos acuáticos depende de la especie vegetal de la que proceda (García, 1995).

Entre los productores primarios se encuentran las algas del perifiton y los briofitos que tapizan los cantos rodados del lecho de la quebrada, viéndose limitado el crecimiento de estos últimos y el de otro tipo de plantas verdes por la velocidad de las corrientes y la escasez de luz (García, 1995) (Fotografía 3).

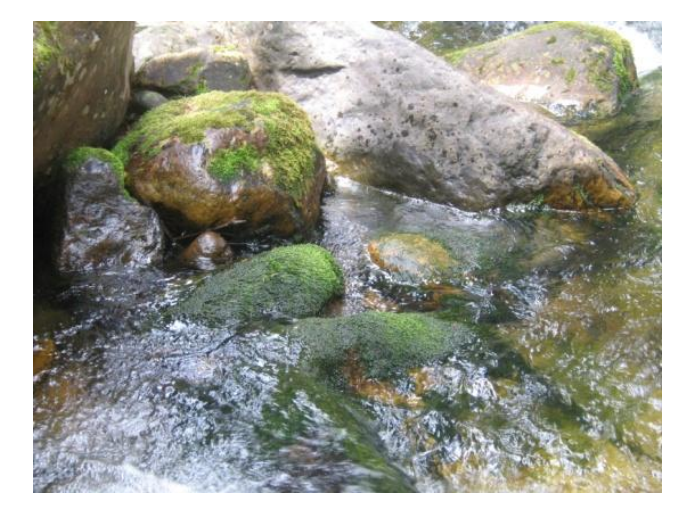

\section{Fotografía 3. Briofitos en las rocas. Quebrada Fucha. Tomada por: Claudia Marcela Rincón Rincón.}

La fauna de insectos acuáticos está bien representada en la quebrada por Ephemeroptera, Trichoptera, Diptera y Lepidoptera.

Con base en la identificación de los contenidos intestinales y morfología de aparatos bucales de las familias de insectos acuáticos más abundantes en la quebrada, se encontraron los grupos funcionales (Tabla 4): filtradores (40\%) que se alimentan de la MOPF, los desmenuzadores (20\%) que se alimentan de MOPG, estos en conjunto poseen mecanismos de alimentación detritívoros $(60 \%)$ y, los raspadores $(30 \%)$ que se alimentan del material vegetal adherido a un sustrato, como perifiton (Merrit, 1996).

La biota acuática es muy diversa (Roldan ,1992) y abundante (García, 1995) en contraposición a la biomasa que es baja, ya que la fauna de insectos acuáticos y de peces está constituida en su mayoría por especies pequeñas (Roldan, 1992). Los dos géneros de desmenuzadores encontrados (Hydroptilidae, Pyralidae) procesan las partículas gruesas del material orgánico, transformándolas en su mayoría en partículas finas y en materia orgánica disuelta; los raspadores (Blepharoceridae, Baetidae, Odontoceridae) aprovechan el perifiton existente; los colectores, fueron el grupo funcional más abundante en el estudio con organismos filtradores y recolectores, quienes se alimentan principalmente filtrando las partículas finas del cestón. (García, 1995). 


\begin{tabular}{|c|c|c|}
\hline \multirow{4}{*}{ Raspador } & Blepharoceridae \\
\cline { 3 - 3 } & & Odontoceridae \\
\cline { 3 - 3 } & & Baetidae \\
\cline { 3 - 3 } & Hydroptilidae \\
\cline { 2 - 3 } & Desmenuzador & Pyralidae \\
\cline { 3 - 3 } & Recolector & Leptophlebiidae \\
\cline { 3 - 3 } & \multirow{4}{*}{ Filtrador } & Chironomidae \\
& & Simulidae \\
\cline { 3 - 3 } & & Ceratopogonidae \\
\cline { 3 - 3 } & & Hydropsychidae \\
\hline
\end{tabular}

Tabla 4. Grupos funcionales

Con relación a la segunda entrada de energía que implica a los productores primarios (perifiton y briófitos), podemos decir a partir del análisis cualitativo que la diversidad de algas es media, encontrando en el muestreo Anabaena, Cosmarium, Eunotia, Mellosira, entre otras (Tabla 2). Esto puede ser reflejo por un lado de las condiciones del sistema (alterado por el cultivo de truchas) y por otro, del valor del oxígeno disuelto, ya que estos organismos son característicos de ambientes con poco grado de contaminación (Pinilla, 1998).

El porcentaje de organismos raspadores $(40 \%)$ da cuenta de que en el sistema la vía principal de energía es la detrítica. Los detritívoros son los primeros en atacar las hojas de árboles recién caídas en el agua que se encuentran asociada a una importante biomasa microbiana. Estos al fragmentar la materia orgánica, producen partículas más finas que derivan a la corriente o se acumulan en algunos puntos del fondo. Por su parte, los invertebrados del grupo de los filtradores, filtran estas partículas así como la biomasa microbiana asociada a ella para alimentarse (Coyne, 2000).

En la quebrada, se evidencia una dominancia de organismos filtradores expresada en larvas de dípteros (Simuliidae y Ceratopogonidae) con 32\% de abundancia cada una.

En cuanto a las poblaciones de peces, se encontró una zona artificial de cultivo de salmónidos (trucha) que vierte bastante material orgánico al sistema (Santander, 2007).

Aunque la cobertura vegetal del dosel sobre la quebrada en el lugar del estudio es alta (65\%), la velocidad de la corriente y la pendiente del sistema arrastran la mayor parte de material depositado, lo cual no permite que gran parte de este pueda ser aprovechado por los organismos presentes.

En síntesis, las relaciones descritas anteriormente se sustentan desde lo propuesto por Vannote et al. (1980), donde expone que en las cabeceras de los ríos la producción primaria es altamente influenciada por la vegetación riparia, que reduce la producción autotrófica 
sustentada con la abundancia y riqueza media de perifiton, y contribuye con grandes cantidades de material alóctono.

En cuanto al reciclaje de nutrientes (espirilización de nutrientes) de la materia orgánica, refuerza la dependencia de las comunidades de los cursos inferiores respecto a las del curso superior, el empleo del material orgánico en deriva por los organismos fijados en el fondo del curso de agua y la reutilización de este material corriente abajo después de la muerte de estos organismos.

Dada la distancia que recorre la quebrada, la altitud y la pendiente $(15 \%)$, la asimilación de nutrientes por parte de la biota se reduce con la velocidad de la corriente, por lo que el material orgánico es reciclado en menor medida, lo que se traduce en menor productividad de la quebrada Fucha en relación con un sistema de zona baja (Santander, 2007).

\section{CONCLUSIONES}

- En la quebrada Fucha se encontraron 4 géneros de insectos acuáticos con una riqueza media de 10 familias, donde las más abundantes son Simuliidae y Ceratopogonidae; presenta una alta equitatividad y baja dominancia. De lo anterior se puede decir que es un sistema con una diversidad media.

- Con base al análisis realizado de la vegetación riparia de la quebrada Fucha y de los contenidos intestinales, se puede decir que la principal fuente de energía aportada al sistema corresponde a la materia orgánica, por lo que se infiere que la vía predominante es la detrítica, caracterizada por el dominio de filtradores y desmenuzadores. Aunque la vía de pastoreo también está presente en la zona en menor medida, esto posiblemente como consecuencia a la mediana abundancia y riqueza de la comunidad perifítica.

- A partir de este estudio se puede considerar que el sistema lótico de la quebrada Fucha, a pesar de tener un alto grado de intervención, posee un número representativo de insectos acuáticos característicos de aguas poco contaminadas.

- Dadas las relaciones existentes en el sistema, puede considerarse que se presentan interacciones poco eficientes, en relación con un sistema de zona baja, respecto al uso de energía expresada en el alto grado de transporte del material, lo que no permite su total aprovechamiento por los diferentes organismos que habitan las zonas altas.

\section{Bibliografía}

- BOTERO B, A. 2004. "El funcionamiento ecológico de un río“. Programa Licenciatura en Biología y Educación Ambiental. Universidad del Quindío. 30 - 39.

- COYNE, M. 2000. "Microbiología del suelo: un enfoque exploratorio". Ediciones Thomson Paraninfo, S.A. Madrid, España. 78 - 219.

- CORFO y DAMA. 2003. “Territorio Río Fucha”. Bogotá, Colombia. 20 - 30.

- CUMMINS, R. 1975. Functional analysis. Journal of Philosophy. 741-765. 
- CUMMINS W, R.; KLUG J, M. 1980. "Fedding ecology of stream invertebrates". 25 (1): 103-132. Web site: www.annualreviews.org/aronline. Fecha última revisión: 20 de Noviembre 2007.

- DONATO, J.; GONZÁLEZ, L. \& RODRÍGUEZ, C. 1996. Ecología de dos sistemas acuáticos de páramo. Academia Colombiana de Ciencias Exactas, Físicas y Naturales. Bogotá, Colombia. 19 (73):259-264.

- GARCIA J. D. \& GONZÁLEZ T .M. 1995. "Restauración de ríos y riberas". Fundación Conde del Valle de Salazar, E.T.S. Ingenieros de Montes Universidad Politécnica de Madrid. España.

- HELLAWELL, J. M. 1986. "Biological indicators of freshwater pollution and environmental management". Elsevier, England.

- LANTHON, M. 2003."Caracterización de macroinvertebrados acuáticos en los ríos Arzobispo y San Cristóbal (Cerros Orientales, Bogotá (Colombia), en la misma franja altitudinal y con énfasis en Chironomidae (Diptera)". Universidad Nacional de Colombia. Tesis de Grado. Bogotá, Colombia.

- MERRIT, R. 1996. "Ecology and distribution of aquatic insects". En: An introduction to the aquatic insects of North America. Kendall Company. Iowa. $74-85$.

- PÉREZ MEDINA, S.; ROMERO BRITO, I. 2005. “Ephemeroptera, Plecoptera Y Trichoptera Del Parque Nacional Natural Cueva De Los Guácharos -Huila- (Quebradas Cristales, Chánchiras y La Cascajosa)". Tesis de Grado. Universidad Pedagógica Nacional. Bogotá, Colombia.

- PINILLA, G. 1998. “Indicadores Biológicos en ecosistemas Acuáticos Continentales de Colombia“. Universidad Jorge Tadeo Lozano, Centro de Investigaciones científicas. Bogotá, Colombia. 31 - 37.

- RAMireZ, A. VIÑA, G. 1998. "Limnología Colombiana. Aportes a su conocimiento y estadísticas de análisis“. Editorial Panamericana. Bogotá, Colombia. 130 - 210.

- RINCÓN, M. E. 1996. “Aspectos bioecólogicos de los Tricópteros de la quebrada Carrizal (Boyacá, Colombia)". Revista Colombiana de Entomología 22 (1): 53 - 60.

- ROLDÁN, P. G. 1988. "Guía para el estudio de los macroinvertebrados acuáticos del departamento de Antioquia." Fondo FEN-Colciencias-Universidad de Antioquia. Bogotá, Colombia. 10 - 217.

- ROLDAN, P. G. 1992. “Fundamentos de Limnología Neotropical”. Editorial Universidad de Antioquia. Medellín, Colombia. 200 - 329.

- ROLDAN, P. G. 2003. “Bioindicación de la calidad del agua en Colombia. Uso del método BMWP/Col". Editorial Universidad de Antioquia. Antioquia, Colombia.

- SANTANDER, M. 2007. "Producción Primaria en Ríos y Arroyos". Facultad de Ciencias Físicas y Matemáticas. Universidad de Chile.

- VANNOTE, R. L., G. W. MINSHALL, K. W. CUMMINS, J. R. SEDELL \& C. E. CUSHING . 1980. "The river continuum concept". Canadian Journal of Fisheries and Aquatic Sciences. 37: 130-137.

- VILLARREAL H., M. ÁlVAREZ, S. CÓRDOBA, F.ESCOBAR, G. FAGUA, F. GAST, H. MENDOZA, M. OSPINA y UMAÑA, A.M. 2006. “Inventarios de biodiversidad. En Manual de métodos para el desarrollo de inventarios de biodiversidad". Segunda 
edición. Programa de Inventarios de Biodiversidad. Instituto de Investigación de Recursos Biológicos Alexander von Humboldt. Bogotá, Colombia.

- ZAPATA, H, R. 2006. "Química de los procesos pedogenéticos”. Universidad Nacional de Colombia. Medellín, Antioquia. 282. 


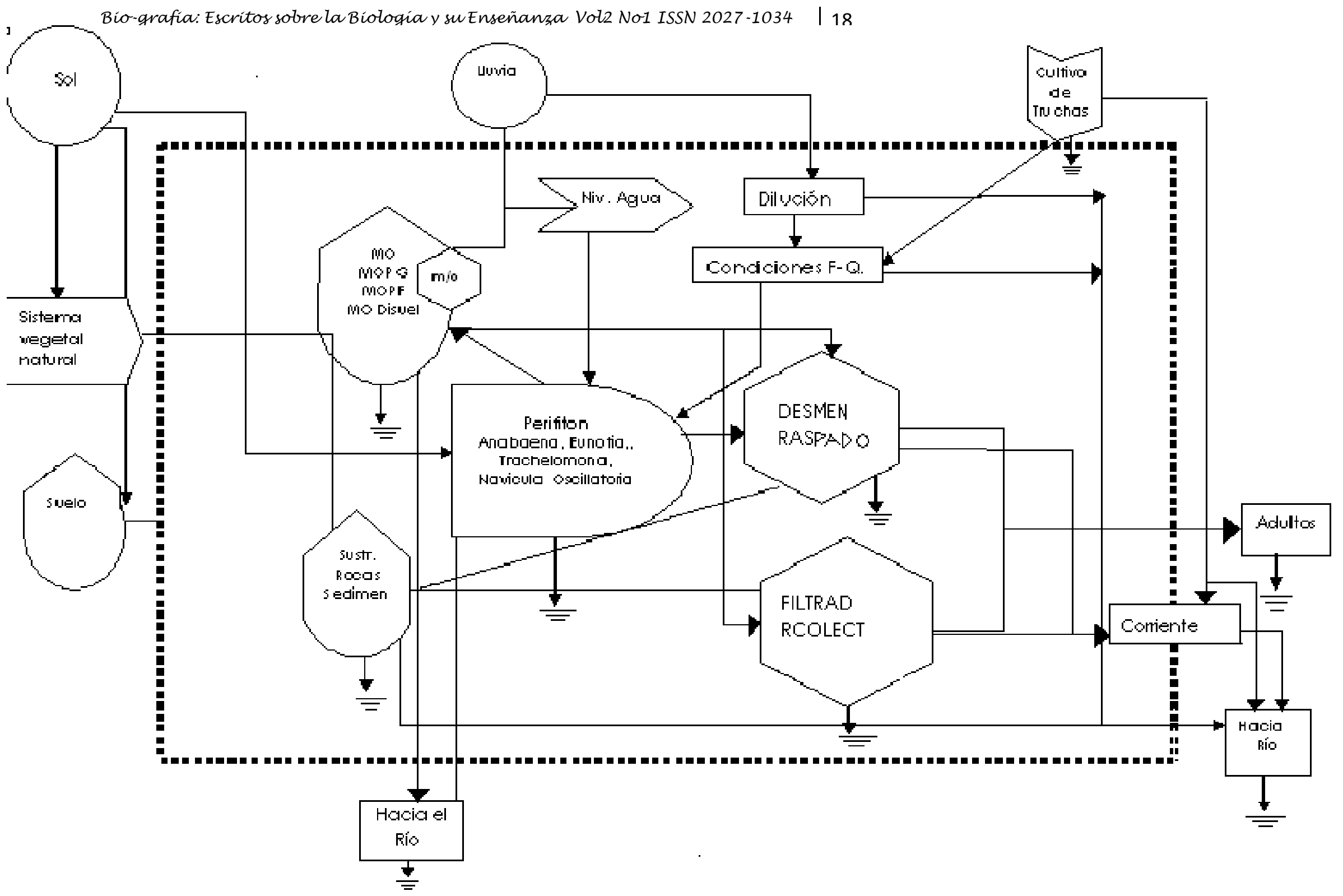

Anexo 1. Diagrama de flujo de energía quebrada Fucha.

Raspadores: Blepharoceridae, Odontoceridae, Baetidae, Hydroptilidae

Desmenuzadores: Pyralidae

Filtradores: Chironomidae, Simuliidae, Ceratopogonidae, Hydropsychidae

Recolectores: Leptophlebiidae 